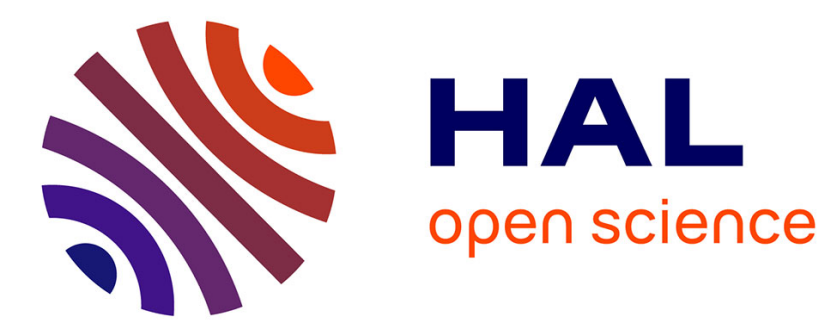

\title{
On the two and three dimensional Oseen potentials
}

Chérif Amrouche, Hamid Bouzit, Ulrich Razafison

\section{To cite this version:}

Chérif Amrouche, Hamid Bouzit, Ulrich Razafison. On the two and three dimensional Oseen potentials. 2010. hal-00549142

\section{HAL Id: hal-00549142 \\ https://hal.science/hal-00549142}

Preprint submitted on 21 Dec 2010

HAL is a multi-disciplinary open access archive for the deposit and dissemination of scientific research documents, whether they are published or not. The documents may come from teaching and research institutions in France or abroad, or from public or private research centers.
L'archive ouverte pluridisciplinaire HAL, est destinée au dépôt et à la diffusion de documents scientifiques de niveau recherche, publiés ou non, émanant des établissements d'enseignement et de recherche français ou étrangers, des laboratoires publics ou privés. 


\title{
On the two and three dimensional Oseen potentials
}

\author{
Chérif Amrouche $^{1}$, Hamid Bouzid ${ }^{1,2}$, Ulrich Razafison ${ }^{3}$ \\ 1 - Université de Pau et des Pays de l'Adour, UMR-CNRS 5142 \\ Laboratoire de Mathématiques Appliquées, \\ IPRA, Avenue de l'Université, 64000 Pau, France \\ Email: cherif.amrouche@univ-pau.fr \\ 2 - Université de Mostaganem, \\ Département de Mathématiques, \\ Algérie, \\ Email: hamidbouzit@yahoo.fr \\ 3 - Université de Franche-Comté, \\ Laboratoire de Mathématiques, CNRS UMR 6623 \\ 16, route de Gray, 25030 Besançon Cedex, France \\ Email: ulrich.razafison@math.cnrs.fr
}

\begin{abstract}
We prove continuity properties for the Oseen potential. As a consequence, we show some new properties on solutions of the Oseen equations. The study relies on weighted Sobolev spaces in order to control the behavior of functions at infinity.
\end{abstract}

Keywords: Riesz potentials, Oseen potentials, Poisson's equations, weighted spaces

AMS Classification: 32A55, 35xx, 35J05

\section{Introduction and Notations}

The purpose of this paper is to establish some new continuity properties in weighted Sobolev spaces for the fundamental solution of Oseen in two and three dimensional. We focus on those operators when they act on $L^{p}$-functions and on distributions that belong to a weighted Sobolev space that will be specified latter. We then use the continuity properties we obtained to find a representation of solutions, to the Oseen equations in $\mathbb{R}^{n}, n=2$ or 3. It is well known that in unbounded domains, it is important to control the behavior of functions at infinity. This is the mathematical reason for dealing with weighted spaces. In previous papers (see for instance [1], [2] and [7]), some weighted inequalities for the 
Oseen potentials are proved under suitable assumptions on the weights. The aim of this work is to improve those results by eventually a modification of the Oseen potentials. The paper is organized as follow. In the next section, we introduce the weighted Sobolev spaces and their main properties for this work. Section 3 is devoted to prove some preliminary results that we will need to establish our main results. Finally in Section 4, we deal with continuity properties of the fundamental solution of Oseen.

We end this section with the Notation that we will use all long this work. We denote by $\mathbb{N}$ the set of all positive integer and $\mathbb{Z}$ the set of all integers. In what follows, $p$ is a real number in the interval $] 1,+\infty[$ and $n$ is an integer number equal to 2 or 3 . The dual exponent of $p$ denoted by $p^{\prime}$ is defined by the relation $\frac{1}{p}+\frac{1}{p^{\prime}}=1$. We will use bold characters for vector or matrix fields. A point in $\mathbb{R}^{n}$ is denoted by $\boldsymbol{x}=\left(x_{1}, \ldots, x_{n}\right)$ and its distance to the origin by

$$
r=|\boldsymbol{x}|=\left(x_{1}^{2}+\ldots+x_{n}^{2}\right)^{1 / 2} .
$$

We denote by $[k]$ the integer part of $k$. For any $\ell \in \mathbb{Z}, \mathbb{P}_{\ell}$ stands for the space of polynomials of degree less than or equal to $\ell$ and $\mathbb{P}_{\ell}^{\Delta}$ the harmonic polynomials of $\mathbb{P}_{\ell}$. If $\ell$ is a negative integer, we set by convention $\mathbb{P}_{\ell}=\{0\}$. We denote by $\mathcal{D}\left(\mathbb{R}^{n}\right)$ the space of $\mathcal{C}^{\infty}$ functions with compact support in $\mathbb{R}^{n}$. We recall that $\mathcal{D}^{\prime}\left(\mathbb{R}^{n}\right)$ is the well known space of distributions and $L^{p}\left(\mathbb{R}^{n}\right)$ is the usual Lebesgue space on $\mathbb{R}^{n}$. For $m \geq 1$, we recall that $W^{m, p}\left(\mathbb{R}^{n}\right)$ is the well-known classical Sobolev spaces. Given a Banach space $B$ with its dual space $B^{\prime}$ and a closed subspace $X$ of $B$, we denote by $B^{\prime} \perp X$ the subspace of $B^{\prime}$ orthogonal to $X$, i.e.,

$$
B^{\prime} \perp X=\left\{f \in B^{\prime}, \quad \forall v \in X,\langle f, v\rangle=0\right\}=(B / X)^{\prime} .
$$

Finally, we will use the symbol $C$ for generic positive constant whose value may change at each occurrence even at the same line.

\section{$2 \quad$ Weighted Sobolev spaces}

We introduce the weight function $\rho(\boldsymbol{x})=\left(1+r^{2}\right)^{1 / 2}$. For a nonnegative integer $m$ and $\alpha \in \mathbb{R}$, we set

$$
k=k(m, n, p, \alpha)= \begin{cases}-1 & \text { if } n / p+\alpha \notin\{1, \ldots, m\} \\ m-n / p-\alpha & \text { if } n / p+\alpha \in\{1, \ldots, m\}\end{cases}
$$

and we define the weighted Sobolev space

$$
\begin{aligned}
W_{\alpha}^{m, p}\left(\mathbb{R}^{n}\right)= & \left\{u \in \mathcal{D}^{\prime}\left(\mathbb{R}^{n}\right) ; \forall \boldsymbol{\lambda} \in \mathbb{N}^{n}, 0 \leq|\boldsymbol{\lambda}| \leq k, \rho^{\alpha-m+|\boldsymbol{\lambda}|}\left(\ln \left(1+\rho^{2}\right)\right)^{-1} \partial^{\boldsymbol{\lambda}} u \in L^{p}\left(\mathbb{R}^{n}\right),\right. \\
& \left.k+1 \leq|\boldsymbol{\lambda}| \leq m, \quad \rho^{\alpha-m+|\boldsymbol{\lambda}|} \partial^{\boldsymbol{\lambda}} u \in L^{p}\left(\mathbb{R}^{n}\right)\right\},
\end{aligned}
$$


which is a Banach space equipped with its natural norm given by

$$
\begin{aligned}
\|u\|_{W_{\alpha}^{m, p}\left(\mathbb{R}^{n}\right)} & =\left(\sum_{0 \leq|\boldsymbol{\lambda}| \leq k}\left\|\rho^{\alpha-m+|\boldsymbol{\lambda}|}\left(\ln \left(1+\rho^{2}\right)\right)^{-1} \partial^{\boldsymbol{\lambda}} u\right\|_{L^{p}\left(\mathbb{R}^{n}\right)}^{p}\right. \\
& \left.+\sum_{k+1 \leq|\boldsymbol{\lambda}| \leq m}\left\|\rho^{\alpha-m+|\boldsymbol{\lambda}|} \partial^{\boldsymbol{\lambda}} u\right\|_{L^{p}\left(\mathbb{R}^{n}\right)}^{p}\right)^{1 / p}
\end{aligned}
$$

We define the semi-norm

$$
|u|_{W_{\alpha}^{m, p}\left(\mathbb{R}^{n}\right)}=\left(\sum_{|\boldsymbol{\lambda}|=m}\left\|\rho^{\alpha} \partial^{\boldsymbol{\lambda}} u\right\|_{L^{p}\left(\mathbb{R}^{n}\right)}^{p}\right)^{1 / p} .
$$

Notice that the logarithmic weight function in the definition of $W_{\alpha}^{m, p}\left(\mathbb{R}^{n}\right)$ only appears for the critical case $n / p+\alpha \in\{1, \ldots, m\}$. We shall now point out some properties of those spaces that will be used all long this paper. For a detailed study we refer to [3] and references therein. All the local properties of the space $W_{\alpha}^{m, p}\left(\mathbb{R}^{n}\right)$ coincide with those of the Sobolev space $W^{m, p}\left(\mathbb{R}^{n}\right)$. The space $\mathcal{D}\left(\mathbb{R}^{n}\right)$ is dense in $W_{\alpha}^{m, p}\left(\mathbb{R}^{n}\right)$. As a consequence, its dual space, denoted by $W_{-\alpha}^{-m, p^{\prime}}\left(\mathbb{R}^{n}\right)$ is a space of distributions. When $n / p+\alpha \notin\{1, \ldots, m\}$, we have the algebraic and topological inclusions

$$
W_{\alpha}^{m, p}\left(\mathbb{R}^{n}\right) \subset W_{\alpha-1}^{m-1, p}\left(\mathbb{R}^{n}\right) \subset \ldots \subset W_{\alpha-m}^{0, p}\left(\mathbb{R}^{n}\right) .
$$

Let $q$ be defined as follow:

$$
\begin{aligned}
& q=[m-n / p-\alpha], \quad \text { if } n / p+\alpha \notin \mathbb{Z}^{-} \\
& q=m-1-n / p-\alpha, \quad \text { otherwise. }
\end{aligned}
$$

Then $\mathbb{P}_{q}$ is the space of all polynomials included in $W_{\alpha}^{m, p}\left(\mathbb{R}^{n}\right)$. Moreover, the following Poincaré-type inequality holds:

$$
\forall u \in W_{\alpha}^{m, p}\left(\mathbb{R}^{n}\right), \quad \inf _{\lambda \in \mathbb{P}_{q^{\prime}}}\|u+\lambda\|_{W_{\alpha}^{m, p}\left(\mathbb{R}^{n}\right)} \leq C|u|_{W_{\alpha}^{m, p}\left(\mathbb{R}^{n}\right)},
$$

where $q^{\prime}=\min (q, 0)$. From $(2.2)$ and the Sobolev's embedding theorems, we have the algebraic and topological identities

$$
W_{0}^{1, p}\left(\mathbb{R}^{n}\right)=\left\{v \in L^{\frac{n p}{n-p}}\left(\mathbb{R}^{n}\right), \quad \nabla v \in L^{p}\left(\mathbb{R}^{n}\right)\right\}, \quad \text { if } 1<p<n
$$

and furthermore for the case $n=3$,

$$
W_{0}^{2, p}\left(\mathbb{R}^{3}\right)=\left\{v \in L^{\frac{3 p}{3-2 p}}\left(\mathbb{R}^{3}\right), \quad \nabla v \in L^{\frac{3 p}{3-p}}\left(\mathbb{R}^{3}\right), \quad \frac{\partial^{2} v}{\partial x_{i} \partial x_{j}} \in L^{p}\left(\mathbb{R}^{3}\right)\right\}, \quad \text { if } 1<p<3 / 2 .
$$




\section{Preliminary results}

Let us recall that the main goal of this work is to prove some new continuity properties for the Oseen potentials by eventually a slight modification of their definition. To that end, we first need to prove some new results on the fundamental solution of the Laplace operator by eventually a modification of their definition. We recall that if $\mathcal{E}$ is the fundamental solution of the Laplace operator, then particularly, for $n=2$, we have $\mathcal{E}(\boldsymbol{x})=\frac{1}{2 \pi} \ln |\boldsymbol{x}|$ and for $n=3, \mathcal{E}(\boldsymbol{x})=-\frac{1}{4 \pi} \frac{1}{|\boldsymbol{x}|}$. Next, for any interval $I \subset \mathbb{R}$, let $1_{I}$, be the function defined by

$$
1_{I}(t)= \begin{cases}1 & \text { if } t \in I, \\ 0 & \text { if } t \notin I .\end{cases}
$$

Now if $f \in L^{p}\left(\mathbb{R}^{n}\right)$, we set

$$
\mathcal{P}_{i} f(\boldsymbol{x})=\left\{\begin{array}{l}
\int_{\mathbb{R}^{3}} \frac{\partial}{\partial x_{i}} \mathcal{E}(\boldsymbol{x}-\boldsymbol{y}) f(\boldsymbol{y}) d \boldsymbol{y}, \quad \text { if } 1<p<n, \\
\int_{\mathbb{R}^{3}}\left(\frac{\partial}{\partial x_{i}} \mathcal{E}(\boldsymbol{x}-\boldsymbol{y})-\frac{\partial}{\partial x_{i}} \mathcal{E}(-\boldsymbol{y})\right) f(\boldsymbol{y}) d \boldsymbol{y} \text { if } p \geq n .
\end{array}\right.
$$

The previous definition will be summarised by the following one:

$$
\mathcal{P}_{i} f(\boldsymbol{x})=\int_{\mathbb{R}^{n}}\left(\frac{\partial}{\partial x_{i}} \mathcal{E}(\boldsymbol{x}-\boldsymbol{y})-1_{[n, \infty[}(p) \frac{\partial}{\partial x_{i}} \mathcal{E}(-\boldsymbol{y})\right) f(\boldsymbol{y}) d \boldsymbol{y} .
$$

For $\boldsymbol{f} \in \boldsymbol{L}^{p}\left(\mathbb{R}^{n}\right)$, we also introduce the operator $\mathcal{P}$ defined by:

$$
\mathcal{P} \boldsymbol{f}(\boldsymbol{x})=\mathcal{P}_{i} f_{i}(\boldsymbol{x})
$$

where the pair of identical indices implies implicit summation. Observe that, if $f \in L^{p}\left(\mathbb{R}^{n}\right)$ with $p \geq n$, then the Riesz potential of first order $I_{1} f$ and also $\frac{\partial \mathcal{E}}{\partial x_{i}} * f$ are not defined. Indeed, let $f$ be a function defined by

$$
f(\boldsymbol{x})=0 \quad \text { if }|\boldsymbol{x}|<1 \quad \text { and } \quad f(\boldsymbol{x})=\frac{1}{|\boldsymbol{x}|} \quad \text { if }|\boldsymbol{x}|>1 .
$$

Then clearly $f \in L^{p}\left(\mathbb{R}^{n}\right)$ if $p>n$ and, for any $|\boldsymbol{x}|<\frac{1}{2}$, we have

$$
I_{1} f(\boldsymbol{x})=\frac{1}{\gamma(1)} \int_{|\boldsymbol{y}|>1} \frac{1}{|\boldsymbol{x}-\boldsymbol{y}|^{n-1}} \frac{1}{|\boldsymbol{y}|} d \boldsymbol{y} \geq \frac{C}{\gamma(1)} \int_{|\boldsymbol{y}|>1} \frac{1}{|\boldsymbol{y}|^{n}} d \boldsymbol{y}=+\infty .
$$

This is the reason of introducing the operator $\mathcal{P}_{i}$. Note that the constants belong to $W_{0}^{1, p}\left(\mathbb{R}^{n}\right)$ if and only if $p \geq n$ (see (2.1)).

Theorem 3.1. The operators

$$
\mathcal{P}_{i}: L^{p}\left(\mathbb{R}^{n}\right) \mapsto W_{0}^{1, p}\left(\mathbb{R}^{n}\right) \quad \text { and } \quad \mathcal{P}: L^{p}\left(\mathbb{R}^{n}\right) \mapsto W_{0}^{1, p}\left(\mathbb{R}^{n}\right)
$$

are contiuous for any $1<p<\infty$. Moreover, we have

$$
\Delta \mathcal{P} \boldsymbol{f}=\operatorname{div} \boldsymbol{f} \text { in } \mathbb{R}^{n} .
$$


Proof. i) Assume first $p<n$. Let $\left(f_{k}\right)_{k \in \mathbb{N}} \subset \mathcal{D}\left(\mathbb{R}^{n}\right)$ be a sequence that tends to $f$ in $L^{p}\left(\mathbb{R}^{n}\right)$. We have

$$
\mathcal{P}_{i} f_{k}(\boldsymbol{x})=I_{2}\left(\frac{\partial f_{k}}{\partial x_{i}}\right),
$$

where $I_{2}$ is the Riesz potential of second order. The above equality implies that $\frac{\partial}{\partial x_{j}} \mathcal{P}_{i} f_{k}=$ $\frac{\partial}{\partial x_{j}} I_{2} \frac{\partial f_{k}}{\partial x_{i}}=R_{i} R_{j} f_{k}$. Thanks to the continuity of the Riesz transform from $L^{p}\left(\mathbb{R}^{n}\right)$ into itself (see [12]), we have

$$
\left\|\frac{\partial}{\partial x_{j}} \mathcal{P}_{i} f_{k}\right\|_{L^{p}\left(\mathbb{R}^{n}\right)} \leq C\left\|f_{k}\right\|_{L^{p}\left(\mathbb{R}^{n}\right)} .
$$

Since $\left|\mathcal{P}_{i} f_{k}(\boldsymbol{x})\right| \leq C\left|I_{1} f_{k}(\boldsymbol{x})\right|$, according to the continuity of the operator $I_{1}: L^{p}\left(\mathbb{R}^{n}\right) \rightarrow$ $L^{q}\left(\mathbb{R}^{n}\right)$ (see [12]), then we have the following estimate:

$$
\left\|\mathcal{P}_{i} f_{k}\right\|_{L^{q}\left(\mathbb{R}^{n}\right)} \leq C\left\|f_{k}\right\|_{L^{p}\left(\mathbb{R}^{n}\right)}, \quad \text { with } \quad \frac{1}{q}=\frac{1}{p}-\frac{1}{n} .
$$

We deduce from (3.7) that

$$
\left\|\frac{\partial}{\partial x_{j}} \mathcal{P}_{i} f\right\|_{L^{p}\left(\mathbb{R}^{n}\right)} \leq C\|f\|_{L^{p}\left(\mathbb{R}^{n}\right)}
$$

Since the norm of $W_{0}^{1, p}\left(\mathbb{R}^{n}\right)$ is equivalent to its semi norm when $p<n$, the statement is proved.

ii) Assume now $p>n$. We set

$$
K(\boldsymbol{x}, \boldsymbol{y})=\frac{\partial}{\partial x_{i}} \mathcal{E}(\boldsymbol{x}-\boldsymbol{y})-\frac{\partial}{\partial x_{i}} \mathcal{E}(-\boldsymbol{y})=C(n)\left(\frac{x_{i}-y_{i}}{|\boldsymbol{x}-\boldsymbol{y}|^{n}}-\frac{-y_{i}}{|\boldsymbol{y}|^{n}}\right) .
$$

Let us show that

$$
\left[\int_{\mathbb{R}^{n}}|K(\boldsymbol{x}, \boldsymbol{y})|^{p^{\prime}} d \boldsymbol{y}\right]^{\frac{1}{p^{\prime}}} \leq C|\boldsymbol{x}|^{1-\frac{n}{p}}
$$

We have:

$$
\left[\int_{\mathbb{R}^{n}}|K(\boldsymbol{x}, \boldsymbol{y})|^{p^{\prime}}\right]^{\frac{1}{p^{\prime}}} \leq\left[\int_{|\boldsymbol{y}|>2|\boldsymbol{x}|}|K(\boldsymbol{x}, \boldsymbol{y})|^{p^{\prime}} d \boldsymbol{y}\right]^{\frac{1}{p^{\prime}}}+\left[\int_{|\boldsymbol{y}|<2|\boldsymbol{x}|}|K(\boldsymbol{x}, \boldsymbol{y})|^{p^{\prime}} d \boldsymbol{y}\right]^{\frac{1}{p^{\prime}}} .
$$

We set

$$
K_{1}=\left[\int_{|\boldsymbol{y}|>2|\boldsymbol{x}|}|K(\boldsymbol{x}, \boldsymbol{y})|^{p^{\prime}} d \boldsymbol{y}\right]^{\frac{1}{p^{\prime}}} \text { and } K_{2}=\left[\int_{|\boldsymbol{y}|<2|\boldsymbol{x}|}|K(\boldsymbol{x}, \boldsymbol{y})|^{p^{\prime}} d \boldsymbol{y}\right]^{\frac{1}{p^{\prime}}} .
$$

For any $\boldsymbol{y}$ such that $|\boldsymbol{y}|>2|\boldsymbol{x}|$, we have, by the means values theorem, the inequality

$$
|K(\boldsymbol{x}, \boldsymbol{y})| \leq C \frac{|\boldsymbol{x}|}{|\boldsymbol{y}|^{n+1}} .
$$

It follows that

$$
K_{1} \leq|\boldsymbol{x}|\left[\int_{|\boldsymbol{y}|>2|\boldsymbol{x}|} \frac{1}{|\boldsymbol{y}|^{n p^{\prime}}} d \boldsymbol{y}\right]^{\frac{1}{p^{\prime}}} \leq C|\boldsymbol{x}|^{1-\frac{n}{p}}
$$


For $K_{2}$, we can write

$$
K_{2} \leq\left[\int_{|\boldsymbol{y}|<2|\boldsymbol{x}|}\left|\frac{x_{i}-y_{i}}{|\boldsymbol{x}-\boldsymbol{y}|^{n}}\right|^{p^{\prime}} d \boldsymbol{y}\right]^{\frac{1}{p^{\prime}}}+\left[\int_{|\boldsymbol{y}|<2|\boldsymbol{x}|}\left|\frac{-y_{i}}{|\boldsymbol{y}|^{n}}\right|^{p^{\prime}} d \boldsymbol{y}\right]^{\frac{1}{p^{\prime}}} .
$$

For any $|\boldsymbol{y}|<2|\boldsymbol{x}|$ we have $|\boldsymbol{x}-\boldsymbol{y}|<3|\boldsymbol{x}|$ and

$$
|K(\boldsymbol{x}, \boldsymbol{y})| \leq C \frac{1}{|\boldsymbol{y}|^{n-1}} .
$$

Then

$$
K_{2} \leq 2\left[\int_{|\boldsymbol{y}|<2|\boldsymbol{x}|} \frac{1}{|\boldsymbol{y}|^{(n-1) p^{\prime}}} d \boldsymbol{y}\right]^{\frac{1}{p^{\prime}}} \leq C|\boldsymbol{x}|^{1-\frac{n}{p}} .
$$

From (3.11) and (3.12) we obtain (3.10). Next from (3.10) and the Hölder's inequality, we get for any $f \in L^{p}\left(\mathbb{R}^{n}\right)$

$$
\left|\mathcal{P}_{i} f(\boldsymbol{x})\right| \leq C|\boldsymbol{x}|^{1-\frac{n}{p}}\|f\|_{L^{p}\left(\mathbb{R}^{n}\right)} .
$$

Let $\left(f_{k}\right)_{k \in \mathbb{N}} \subset \mathcal{D}\left(\mathbb{R}^{n}\right)$ be a sequence that tends to $f$ in $L^{p}\left(\mathbb{R}^{n}\right)$. We have

$$
\mathcal{P}_{i} f_{k}(\boldsymbol{x})=I_{2}\left(\frac{\partial f_{k}}{\partial x_{i}}\right)-C \int_{\operatorname{Supp} f_{k}} \frac{y_{i}}{|\boldsymbol{y}|^{n-1}} f_{k}(\boldsymbol{y}) d \boldsymbol{y} .
$$

This implies that $\frac{\partial}{\partial x_{j}} \mathcal{P}_{i} f_{k}=\frac{\partial}{\partial x_{j}} I_{2} \frac{\partial f_{k}}{\partial x_{i}}$. As in the first case, $\frac{\partial}{\partial x_{j}} \mathcal{P}_{i} f_{k}$ tends to $\frac{\partial}{\partial x_{j}} I_{2} \frac{\partial f}{\partial x_{i}}$ in $L^{p}\left(\mathbb{R}^{n}\right)$. On the other hand, from (3.13), $\mathcal{P}_{i} f_{k}$ tends to $I_{2} \frac{\partial f}{\partial x_{i}}$ in $W_{-1-\varepsilon}^{0, p}\left(\mathbb{R}^{n}\right)$, for any $\varepsilon>0$. Thus we deduce that $\frac{\partial}{\partial x_{j}} \mathcal{P}_{i} f=\frac{\partial}{\partial x_{j}} I_{2} \frac{\partial f}{\partial x_{i}}$ and

$$
\left\|\frac{\partial}{\partial x_{j}} \mathcal{P}_{i} f\right\|_{L^{p}\left(\mathbb{R}^{n}\right)} \leq C\|f\|_{L^{p}\left(\mathbb{R}^{n}\right)}
$$

Besides, since $\mathcal{P}_{i} f(\mathbf{0})=0$, we have (see [3] Lemma 3.2)

$$
\left\|\mathcal{P}_{i} f\right\|_{W_{-1}^{0, p}\left(\mathbb{R}^{n}\right)} \leq\left\|\frac{\partial}{\partial x_{j}} \mathcal{P}_{i} f\right\|_{L^{p}\left(\mathbb{R}^{n}\right)} \leq C\|f\|_{L^{p}\left(\mathbb{R}^{n}\right)},
$$

whith implies that the operator $\mathcal{P}_{i}: L^{p}\left(\mathbb{R}^{n}\right) \rightarrow W_{0}^{1, p}\left(\mathbb{R}^{n}\right)$ is continuous.

iii) Finally, for $p=n$, we show that

$$
\int_{\mathbb{R}^{n}}|K(\boldsymbol{x}, \boldsymbol{y})|^{\frac{n}{n-1}} d \boldsymbol{y} \leq C(1+\operatorname{Ln}(2+|\boldsymbol{x}|))
$$

and we proceed as previousely to prove the statement.

Next for $f \in L^{p}\left(\mathbb{R}^{n}\right)$, we introduce the operator

$$
J_{2} f(\boldsymbol{x})=\int_{\mathbb{R}^{3}}\left(\mathcal{E}(\boldsymbol{x}-\boldsymbol{y})-1_{\left[\frac{n}{2}, \infty[\right.}(p) \mathcal{E}(-\boldsymbol{y})-1_{[n, \infty[}(p) \boldsymbol{x} . \nabla \mathcal{E}(-\boldsymbol{y})\right) f(\boldsymbol{y}) d \boldsymbol{y} .
$$

By proceeding as for Theorem 3.1, we prove the following result. 
Theorem 3.2. The operator $J_{2}: L^{p}\left(\mathbb{R}^{n}\right) \mapsto W_{0}^{2, p}\left(\mathbb{R}^{n}\right)$ is continuous for any $1<p<\infty$. Moreover, if $f \in \boldsymbol{L}^{p}\left(\mathbb{R}^{n}\right)$, then the solutions of the Poisson's equation

$$
-\Delta u=f \quad \text { in } \mathbb{R}^{n}
$$

are in the form

$$
u=J_{2} f+\lambda, \quad \lambda \in \mathbb{P}_{[2-n / p]} .
$$

\section{The two and three dimensional Oseen potentials}

In this section, we consider the Oseen problem in $\mathbb{R}^{n}$ :

$$
\begin{array}{r}
-\Delta \boldsymbol{u}+k \frac{\partial \boldsymbol{u}}{\partial x_{1}}+\nabla \pi=\boldsymbol{f} \text { in } \mathbb{R}^{n}, \\
\operatorname{div} \boldsymbol{u}=g \text { in } \mathbb{R}^{n},
\end{array}
$$

where $k>0$. For the investigation of (4.15) in weighted Sobolev spaces, we refer to [8], [9], [5], [7], [11] and [2]. Further works can be found in [6].

We first recall briefly the definition of the fundamental of Oseen by following [10]. We denote $(\mathcal{O}, \boldsymbol{e})$ the fundamental solution of Oseen defined by

$$
\begin{gathered}
\mathcal{O}_{i j}(\boldsymbol{x}, \boldsymbol{y})=\left(\delta_{i j} \Delta-\frac{\partial^{2}}{\partial y_{i} \partial y_{j}}\right) \Phi(\boldsymbol{x}, \boldsymbol{y}) \\
e_{j}(\boldsymbol{x}, \boldsymbol{y})=-\frac{\partial}{\partial y_{j}}\left(\Delta+k \frac{\partial}{\partial y_{1}}\right) \Phi(\boldsymbol{x}, \boldsymbol{y}),
\end{gathered}
$$

where $i, j=1, \ldots n$ and $\Phi(\boldsymbol{x}, \boldsymbol{y})$ is a smooth function for $\boldsymbol{x} \neq \boldsymbol{y}$. In order to have (4.16) a singular solution to $(4.15)$, we require that

$$
\Delta\left(\Delta+k \frac{\partial}{\partial y_{1}}\right) \Phi(\boldsymbol{x}, \boldsymbol{y})=\Delta \mathcal{E}(|\boldsymbol{x}-\boldsymbol{y}|),
$$

where we recall that $\mathcal{E}$ is the fundanental solution to the Laplace equation. We search the solution to (4.17) in the form

$$
\begin{array}{r}
\Phi(\boldsymbol{x}, \boldsymbol{y})=\frac{1}{k} \int^{y_{1}-x_{1}}\left[\Phi_{2}\left(\tau, y_{2}-x_{2}, \ldots, y_{n}-x_{n}\right)\right. \\
\left.-\Phi_{1}\left(\tau, y_{2}-x_{2}, \ldots, y_{n}-x_{n}\right)\right] d \tau,
\end{array}
$$

where $\Phi_{1}$ and $\Phi_{2}$ must be selected suitably. From (4.17), we formally get

$$
\Delta\left(\Delta+k \frac{\partial}{\partial y_{1}}\right)\left(\Phi_{2}-\Phi_{1}\right)=k \Delta\left(\frac{\partial \mathcal{E}}{\partial y_{1}}\right) .
$$

Choosing $\Phi_{2}(\boldsymbol{x}, \boldsymbol{y})=\mathcal{E}(|\boldsymbol{x}-\boldsymbol{y}|)$, it is sufficient to take

$$
\left(\Delta+k \frac{\partial}{\partial y_{1}}\right) \Phi_{1}=\Delta \mathcal{E}
$$


Moreover, from (4.17) and the second equation of (4.16), we have

$$
e_{j}(\boldsymbol{x}, \boldsymbol{y})=-\frac{\partial}{\partial y_{j}} \mathcal{E}(|\boldsymbol{x}-\boldsymbol{y}|)
$$

We take

$$
\Phi_{1}(\boldsymbol{x}, \boldsymbol{y})=\frac{e^{-\frac{k}{2}\left(y_{1}-x_{1}\right)}}{|\boldsymbol{x}-\boldsymbol{y}|^{\frac{n-2}{2}}} f(\lambda|\boldsymbol{x}-\boldsymbol{y}|)
$$

and we deduce

$$
\begin{aligned}
\left(\Delta+k \frac{\partial}{\partial y_{1}}\right) \Phi_{1}(\boldsymbol{x}, \boldsymbol{y}) & =\frac{e^{-\frac{k}{2}\left(y_{1}-x_{1}\right)}}{|\boldsymbol{x}-\boldsymbol{y}|^{\frac{n+2}{2}}}\left[z^{2} f^{\prime \prime}(z)+z f^{\prime}(z)-\left(\left[\frac{n-2}{2}\right]^{2}+z^{2}\right) f(z)\right] \\
& \equiv \frac{e^{-\frac{k}{2}\left(y_{1}-x_{1}\right)}}{|\boldsymbol{x}-\boldsymbol{y}|^{\frac{n+2}{2}}} \mathcal{L}(f)
\end{aligned}
$$

where $z=\frac{k}{2}|\boldsymbol{x}-\boldsymbol{y}|$ and the prime denotes differentiation with respect to $z$. The equation $\mathcal{L}(f)=0$ is the Bessel's modified equation which admits two independent solutions $I_{\frac{n-2}{2}}(z)$ and $K_{\frac{n-2}{2}}(z)$ called modified Bessel's functions. We have for $z>0$

$$
\begin{aligned}
K_{0}(z) & =-\ln z+\ln 2-\gamma-\ln \left(\frac{z}{2}\right) \sum_{k=1}^{\infty} \frac{1}{(k !)^{2}}\left(\frac{z}{2}\right)^{2 k} \\
& +\sum_{k=1}^{\infty} \frac{1}{(k !)^{2}}\left(\sum_{j=1}^{k}\left(\frac{1}{j}\right)-\gamma\right)\left(\frac{z}{2}\right)^{2 k} \text { if } n=2 \\
K_{\frac{1}{2}}(z) & =\left(\frac{\pi}{2 z}\right)^{1 / 2} e^{-z} \text { if } n=3 .
\end{aligned}
$$

Since $K_{\frac{1}{2}}$ has a simpler form than $K_{0}$, we shall only give here the expression of $\Phi$ when $n=3$. From (4.21) and (4.24), we have

$$
\Phi_{1}=-\frac{1}{2 \pi}\left(\frac{k}{4 \pi|\boldsymbol{x}-\boldsymbol{y}|}\right)^{1 / 2} K_{\frac{1}{2}}\left(\frac{k}{2}|\boldsymbol{x}-\boldsymbol{y}|\right) e^{-k\left(y_{1}-x_{1}\right) / 2} .
$$

Inserting (4.25) to (4.18),

$$
\Phi(\boldsymbol{x}-\boldsymbol{y})=\frac{1}{4 \pi k} \int^{y_{1}-x_{1}} \frac{1-\exp \left\{-\frac{k}{2}\left[\sqrt{\tau^{2}+\left(x_{2}-y_{2}\right)^{2}+\left(x_{3}-y_{3}\right)^{2}}+\tau\right]\right\}}{\sqrt{\tau^{2}+\left(x_{2}-y_{2}\right)^{2}+\left(x_{3}-y_{3}\right)^{2}}} d \tau .
$$

We may now fix the constant up to which $\Phi$ is defined by taking $\Phi(0)=0$. Therefore, when $n=3$, the fundamental velocity $\mathcal{O}$ can be written in the form:

$$
\mathcal{O}_{i j}(\boldsymbol{x})=\left(\delta_{i j} \Delta-\frac{\partial}{\partial x_{i}} \frac{\partial}{\partial x_{j}}\right) \Phi(\boldsymbol{x})
$$

where

$$
\Phi(\boldsymbol{x})=\frac{1}{4 \pi k} \int_{0}^{k s(\boldsymbol{x}) / 2} \frac{1-e^{-t}}{t} d t
$$


Observe that $\mathcal{O}_{i j} \in L^{p}\left(\mathbb{R}^{3}\right)$ if $2<p<3$ and $\nabla \mathcal{O}_{i j} \in \boldsymbol{L}^{p}\left(\mathbb{R}^{3}\right)$ if $4 / 3<p<3 / 2$ (see for instance [1] and [7]). Next, since in this work, we are really interested in the asymptotic behavior of the fundamental solution of Oseen, we shall give such properties when $n=2$. the behavior of the tensor $\mathcal{O}$ for $r \rightarrow 0$ is given by:

$$
\mathcal{O}_{i j}(\boldsymbol{x})=\frac{1}{4 \pi}\left(\delta_{i j} \ln r+\frac{x_{i} x_{j}}{r^{2}}\right)+o(1) .
$$

Now let $r \rightarrow \infty$, then we have:

$$
\begin{aligned}
& \mathcal{O}_{11}(\boldsymbol{x})=-\frac{\cos \theta}{2 \pi r}+\frac{1}{4 \sqrt{\pi r}} e^{-s(\boldsymbol{x}) / 2}\left[1+\cos \theta+\frac{1}{4 r}(3 \cos \theta-1)+\sigma(r)\right], \\
& \mathcal{O}_{12}(\boldsymbol{x})=\mathcal{O}_{21}(\boldsymbol{x})=-\frac{\sin \theta}{2 \pi r}+\frac{\sin \theta}{4 \sqrt{\pi r}} e^{-s(\boldsymbol{x}) / 2}\left[1+\frac{3}{4 r}+\sigma(r)\right], \\
& \mathcal{O}_{22}(\boldsymbol{x})=-\frac{\cos \theta}{2 \pi r}+\frac{1}{4 \sqrt{\pi r}} e^{-s(\boldsymbol{x}) / 2}\left[-\frac{s(\boldsymbol{x})}{2}+\frac{1}{4 r}(1+3 \cos \theta)+\sigma(r)\right],
\end{aligned}
$$

where

$$
\frac{\mathrm{d}^{k} \sigma}{\mathrm{d} r^{k}}=O\left(r^{-2-k}\right) \quad \text { as } r \rightarrow \infty, k \geq 0 \text { and } s(\boldsymbol{x})=r-x_{1} .
$$

We have the summability properties (see $[2]) \mathcal{O}_{2 j} \in L^{p}\left(\mathbb{R}^{2}\right)$ if $p>2$ and $\mathcal{O}_{11} \in L^{p}\left(\mathbb{R}^{2}\right)$ if $p>3$. Note that the fundamental pressure $\boldsymbol{e}$ is given by

$$
e_{j}(\boldsymbol{x})=-\frac{\partial \mathcal{E}(\boldsymbol{x})}{\partial x_{j}}=\frac{1}{2(n-1) \pi} \frac{x_{j}}{r^{n}}, j=1, \ldots, n .
$$

Now it also well known that if the data $(f, g) \in \mathcal{D}\left(\mathbb{R}^{n}\right) \times \mathcal{D}\left(\mathbb{R}^{n}\right)$, then the Oseen problem (4.15) has an explicit solution $\left(\boldsymbol{u}^{*}, \pi^{*}\right) \in \mathcal{C}^{\infty}\left(\mathbb{R}^{n}\right) \times \mathcal{C}^{\infty}\left(\mathbb{R}^{n}\right)$ defined by

$$
\begin{aligned}
& u_{i}^{*}=\mathcal{O}_{i j} * f_{j}+\frac{\partial \mathcal{E}}{\partial x_{i}} * g \\
& \pi^{*}=\frac{\partial \mathcal{E}}{\partial x_{j}} * f_{j}+g-\frac{\partial \mathcal{E}}{\partial x_{1}} * g .
\end{aligned}
$$

It is now natural to inquire about the validity of (4.27) if $\boldsymbol{f} \in \boldsymbol{L}^{p}\left(\mathbb{R}^{n}\right)$ and if $g$ belongs to a subspace of $W_{0}^{1, p}\left(\mathbb{R}^{n}\right)$ that will be specified in the remaining of the paper. For convenience, we introduce the notation $\mathcal{O} * \boldsymbol{f}$ which denotes the vector field defined by $\mathcal{O}_{i j} * f_{j}, i=1, \ldots, n$. We first have the following properties.

Proposition 4.1. Let $\boldsymbol{f} \in \boldsymbol{L}^{p}\left(\mathbb{R}^{n}\right)$. Then $\frac{\partial^{2} \mathcal{O}}{\partial x_{i} \partial x_{j}} * \boldsymbol{f} \in \boldsymbol{L}^{p}\left(\mathbb{R}^{n}\right)$ and $\frac{\partial \mathcal{O}}{\partial x_{1}} * \boldsymbol{f} \in \boldsymbol{L}^{p}\left(\mathbb{R}^{n}\right)$ for any $1<p<\infty$, where $\frac{\partial^{2} \mathcal{O}}{\partial x_{i} \partial x_{j}} * \boldsymbol{f} \in \boldsymbol{L}^{p}\left(\mathbb{R}^{n}\right)$ and $\frac{\partial \mathcal{O}}{\partial x_{1}} * \boldsymbol{f} \in \boldsymbol{L}^{p}\left(\mathbb{R}^{n}\right)$ are defined in the sense of principal value, and we have the estimate

$$
\left\|\frac{\partial^{2} \mathcal{O}}{\partial x_{i} \partial x_{j}} * \boldsymbol{f}\right\|_{\boldsymbol{L}^{p}\left(\mathbb{R}^{n}\right)}+\left\|\frac{\partial \mathcal{O}}{\partial x_{1}} * \boldsymbol{f}\right\|_{\boldsymbol{L}^{p}\left(\mathbb{R}^{n}\right)} \leq C\|\boldsymbol{f}\|_{\boldsymbol{L}^{p}\left(\mathbb{R}^{n}\right)} .
$$

Moreover, 
a) let $n=2$, then $\nabla \mathcal{O}_{2 j} * f_{j} \in L^{p}\left(\mathbb{R}^{n}\right)$ and

(i) if $1<p<2$, then $\mathcal{O}_{2 j} * f_{j} \in L^{\frac{2 p}{2-p}}\left(\mathbb{R}^{2}\right) \cap L^{\infty}\left(\mathbb{R}^{2}\right)$,

(ii) if $1<p<3 / 2$, then $\mathcal{O}_{11} * f_{1} \in L^{\frac{3 p}{3-2 p}}\left(\mathbb{R}^{2}\right) \cap L^{\infty}\left(\mathbb{R}^{2}\right)$.

b) Let $n=3$, then

(i) $\mathcal{O} * \boldsymbol{f} \in \boldsymbol{L}^{\frac{3 p}{3-2 p}}\left(\mathbb{R}^{n}\right)$ if $1<p<3 / 2$ and $\mathcal{O} * \boldsymbol{f} \in \boldsymbol{L}^{\frac{2 p}{2-p}}\left(\mathbb{R}^{n}\right)$ if $1<p<2$,

(ii) $\nabla \mathcal{O} * \boldsymbol{f} \in \boldsymbol{L}^{\frac{3 p}{3-p}}\left(\mathbb{R}^{n}\right)$ if $1<p<3$ and $\nabla \mathcal{O} * \boldsymbol{f} \in \boldsymbol{L}^{\frac{4 p}{4-p}}\left(\mathbb{R}^{n}\right)$ if $1<p<4$.

In all the cases, we have the corresponding estimates.

The proof of the above proposition can be found in [2] when $n=2$. For $n=3$ it is proved by combining results proved in [1] and [7].

Note that from the previous proposition, for $n=2$, the operator $\boldsymbol{f} \mapsto \mathcal{O}_{2 j} * f_{j}$ from $\boldsymbol{L}^{p}\left(\mathbb{R}^{2}\right)$ into $L^{\frac{2 p}{2-p}}\left(\mathbb{R}^{2}\right) \cap L^{\infty}\left(\mathbb{R}^{2}\right)$ is continuous if $1<p<2$ and the operator $\boldsymbol{f} \mapsto \mathcal{O}_{11} * f_{1}$ from $\boldsymbol{L}^{p}\left(\mathbb{R}^{2}\right)$ into $L^{\frac{3 p}{3-2 p}}\left(\mathbb{R}^{2}\right) \cap L^{\infty}\left(\mathbb{R}^{2}\right)$ is continuous if $1<p<3 / 2$. For $n=3$, the operators $\boldsymbol{f} \mapsto \mathcal{O} * \boldsymbol{f}$ from $\boldsymbol{L}^{p}\left(\mathbb{R}^{3}\right)$ into $\boldsymbol{L}^{\frac{2 p}{2-p}}\left(\mathbb{R}^{3}\right)$ and $\boldsymbol{f} \mapsto \nabla \mathcal{O} * \boldsymbol{f}$ from $\boldsymbol{L}^{p}\left(\mathbb{R}^{3}\right)$ into $\boldsymbol{L}^{\frac{4 p}{4-p}}\left(\mathbb{R}^{3}\right)$ are continuous if $1<p<2$. Thus we observe that if $\boldsymbol{f} \in \boldsymbol{L}^{p}\left(\mathbb{R}^{n}\right)$, then the explicit forms (4.27) are not necessarily defined for any $1<p<\infty$. This is the reason of introducing the modified operator $\mathcal{O}$ defined by for $\boldsymbol{f} \in \boldsymbol{L}^{p}\left(\mathbb{R}^{n}\right)$ :

- If $n=2$, then $\mathcal{O}=\left(\mathcal{O}_{1}, \mathcal{O}_{2}\right)$, where

$$
\begin{aligned}
& O_{1} \boldsymbol{f}(\boldsymbol{x})=\int_{\mathbb{R}^{2}}\left[\mathcal{O}_{1 j}(\boldsymbol{x}-\boldsymbol{y})-1_{[3 / 2,+\infty}\left[(p) \mathcal{O}_{1 j}(-\boldsymbol{y})-1_{[3,+\infty}(p) x_{2} \frac{\partial}{\partial x_{2}} \mathcal{O}_{1 j}(-\boldsymbol{y})\right] f_{j}(\boldsymbol{x}) d \boldsymbol{y},\right. \\
& \mathcal{O}_{2} \boldsymbol{f}(\boldsymbol{x})=\int\left[\mathcal{O}_{2 j}(\boldsymbol{x}-\boldsymbol{y})-1_{[2,+\infty[}(p) \mathcal{O}_{2 j}(-\boldsymbol{y})\right] f_{j}(\boldsymbol{x}) d \boldsymbol{y} .
\end{aligned}
$$

- If $n=3$, then

$$
\begin{aligned}
(\mathcal{O} f(\boldsymbol{x}))_{i} & =\mathcal{O}_{i j} f_{j}(\boldsymbol{x}) \\
& =\int_{\mathbb{R}^{3}}\left(\mathcal{O}_{i j}(\boldsymbol{x}-\boldsymbol{y})-1_{[2, \infty[}(p) \mathcal{O}_{i j}(-\boldsymbol{y})-1_{[4, \infty[}(p) \boldsymbol{x}^{\prime} \cdot \nabla^{\prime} \mathcal{O}_{i j}(-\boldsymbol{y})\right) f_{j}(\boldsymbol{y}) d \boldsymbol{y},
\end{aligned}
$$

where $\boldsymbol{x}^{\prime}=\left(0, x_{2}, x_{3}\right)$ and $\nabla^{\prime}=\left(0, \frac{\partial}{\partial x_{2}}, \frac{\partial}{\partial x_{3}}\right)$.

Let us now introduce the following anisotropically weighted spaces

$$
\begin{aligned}
X_{p}\left(\mathbb{R}^{n}\right) & =\left\{v \in L^{p}\left(\mathbb{R}^{n}\right), \frac{\partial v}{\partial x_{1}} \in W_{0}^{-2, p}\left(\mathbb{R}^{n}\right)\right\}, \\
Y_{0}^{1, p}\left(\mathbb{R}^{n}\right) & =\left\{v \in W_{0}^{1, p}\left(\mathbb{R}^{n}\right), \frac{\partial v}{\partial x_{1}} \in W_{0}^{-1, p}\left(\mathbb{R}^{n}\right)\right\}
\end{aligned}
$$


and

$$
Z_{0}^{2, p}\left(\mathbb{R}^{n}\right)=\left\{v \in W_{0}^{2, p}\left(\mathbb{R}^{n}\right), \frac{\partial v}{\partial x_{1}} \in L^{p}\left(\mathbb{R}^{n}\right)\right\} .
$$

These are Banach spaces when endowed respectively with the norms

$$
\begin{gathered}
\|v\|_{X_{p}\left(\mathbb{R}^{n}\right)}=\|v\|_{L^{p}\left(\mathbb{R}^{n}\right)}+\left\|\frac{\partial v}{\partial x_{1}}\right\|_{W_{0}^{-2, p}\left(\mathbb{R}^{n}\right)}, \\
\|v\|_{Y_{0}^{1, p}\left(\mathbb{R}^{n}\right)}=\|v\|_{W_{0}^{1, p}\left(\mathbb{R}^{n}\right)}+\left\|\frac{\partial v}{\partial x_{1}}\right\|_{W_{0}^{-1, p}\left(\mathbb{R}^{n}\right)}
\end{gathered}
$$

and

$$
\|v\|_{Z_{0}^{2, p}\left(\mathbb{R}^{n}\right)}=\|v\|_{W_{0}^{2, p}\left(\mathbb{R}^{n}\right)}+\left\|\frac{\partial v}{\partial x_{1}}\right\|_{L^{p}\left(\mathbb{R}^{n}\right)} .
$$

Proceeding now as in Theorem 3.1, we have the following result.

Theorem 4.2. The operator

$$
\mathcal{O}: \boldsymbol{L}^{p}\left(\mathbb{R}^{n}\right) \mapsto Z_{0}^{2, p}\left(\mathbb{R}^{n}\right)
$$

is continuous for $1<p<\infty$.

In order to give an explicit form for the solutions of the Oseen problem (4.15), when the data $(\boldsymbol{f}, g)$ do belong to the space $\boldsymbol{L}^{p}\left(\mathbb{R}^{n}\right) \times Y_{0}^{1, p}\left(\mathbb{R}^{n}\right)$, we need the following preliminary lemma.

Lemma 4.3. Assume $g \in Y_{0}^{1, p}\left(\mathbb{R}^{n}\right)$ and let $\mathcal{P}_{j}$ be defined by (3.5).

(i) If $1<p<n$, then $\nabla \mathcal{P}_{j} g \in \mathbf{Y}_{0}^{1, p}\left(\mathbb{R}^{n}\right) \cap \boldsymbol{W}_{0}^{1, \frac{n p}{n-p}}\left(\mathbb{R}^{n}\right), \mathcal{P}_{j} g \in Z_{0}^{2, p}\left(\mathbb{R}^{n}\right)$ and we have the estimate

$$
\left\|\nabla \mathcal{P}_{j} g\right\|_{\mathbf{Y}_{0}^{1, p}\left(\mathbb{R}^{n}\right)}+\left\|\nabla \mathcal{P}_{j} g\right\|_{\mathbf{Y}_{0}^{1, \frac{n p}{n-p}\left(\mathbb{R}^{n}\right)}}+\left\|\mathcal{P}_{j} g\right\|_{Z_{0}^{2, p}\left(\mathbb{R}^{n}\right)} \leq C\|g\|_{Y_{0}^{1, p}\left(\mathbb{R}^{n}\right)} .
$$

Additionally, if $n=3$ and $1<p<3 / 2$, then $\mathcal{P}_{j} g \in L^{\frac{3 p}{3-2 p}}\left(\mathbb{R}^{3}\right)$ and the following estimate holds

$$
\left\|\mathcal{P}_{j} g\right\|_{L^{3-2 p}\left(\mathbb{R}^{3}\right)} \leq C\|g\|_{Y_{0}^{1, p}\left(\mathbb{R}^{3}\right)} .
$$

(ii) If $p \geq n$, then $J_{2} \frac{\partial g}{\partial x_{j}} \in Z_{0}^{2, p}\left(\mathbb{R}^{n}\right)$, where the operator $J_{2}$ is defined by (3.14), and we have

$$
\left\|J_{2} \frac{\partial g}{\partial x_{j}}\right\|_{Z_{0}^{2, p}\left(\mathbb{R}^{n}\right)} \leq C\|g\|_{Y_{0}^{1, p}\left(\mathbb{R}^{n}\right)} .
$$

Proof. (i) If $1<p<n$, then $g \in Y_{0}^{1, p}\left(\mathbb{R}^{n}\right)$ implies that $g \in L^{\frac{n p}{n-p}}\left(\mathbb{R}^{3}\right)$ (see (2.3)). Thanks to Theorem 3.1, $\mathcal{P}_{j} g \in W_{0}^{1, \frac{n p}{n-p}}\left(\mathbb{R}^{n}\right)$ and we have

$$
\left\|\mathcal{P}_{j} g\right\|_{W_{0}^{1, \frac{n p}{n-p}}\left(\mathbb{R}^{n}\right)} \leq C\|g\|_{Y_{0}^{1, p}\left(\mathbb{R}^{n}\right)} .
$$


Besides, due to the fact that $\nabla g \in L^{p}\left(\mathbb{R}^{n}\right)$, we also have $\mathcal{P}_{j} \nabla g \in W_{0}^{1, p}\left(\mathbb{R}^{n}\right)$. Now, let $\left(g_{k}\right)_{k \in \mathbb{N}} \in \mathcal{D}\left(\mathbb{R}^{n}\right)$ be a sequence that tends to $g$ in $Y_{0}^{1, p}\left(\mathbb{R}^{n}\right)$. We know that we have

$$
\frac{\partial}{\partial x_{1}} \mathcal{P}_{j} g_{k}=\mathcal{P}_{j} \frac{\partial g_{k}}{\partial x_{1}}
$$

Moreover, since $1<p<n$, then $g_{k}$ tends to $g$ in $L^{\frac{n p}{n-p}}\left(\mathbb{R}^{3}\right)$ which implies that $\mathcal{P}_{j} g_{k}$ tends to $\mathcal{P}_{j} g$ in $W_{0}^{1, \frac{n p}{n-p}}\left(\mathbb{R}^{3}\right)$, in particular $\frac{\partial}{\partial x_{1}} \mathcal{P}_{j} g_{k}$ tends to $\frac{\partial}{\partial x_{1}} \mathcal{P}_{j} g$ in $L^{\frac{n p}{n-p}}\left(\mathbb{R}^{3}\right)$. Besides, since $\frac{\partial g_{k}}{\partial x_{1}}$ tends to $\frac{\partial g}{\partial x_{1}}$ in $W_{0}^{-1, p}\left(\mathbb{R}^{n}\right)$, then $\mathcal{P}_{j} \frac{\partial g_{k}}{\partial x_{1}}$ tends to $\mathcal{P}_{j} \frac{\partial g}{\partial x_{1}}$ in $L^{p}\left(\mathbb{R}^{n}\right)$. This implies that $\frac{\partial}{\partial x_{1}} \mathcal{P}_{j} g=\mathcal{P}_{j} \frac{\partial g}{\partial x_{1}}$. By the same way, we prove that $\nabla \mathcal{P}_{j} g=\mathcal{P}_{j} \nabla g$. Thus, we deduce that $\nabla \mathcal{P}_{j} g \in \boldsymbol{W}_{0}^{1, p}\left(\mathbb{R}^{n}\right)$ and $\frac{\partial}{\partial x_{1}} \mathcal{P}_{j} g \in L^{p}\left(\mathbb{R}^{n}\right)$. Thus, we have that $\mathcal{P}_{j} g \in Z_{0}^{2, p}\left(\mathbb{R}^{3}\right)$, $\nabla \mathcal{P}_{j} g \in \mathbf{Y}_{0}^{1, p}\left(\mathbb{R}^{3}\right)$ and the estimates (4.28) and (4.29) hold. Additionally if $n=3$ and $1<p<3 / 2$, then $\nabla \mathcal{P}_{j} g \in \boldsymbol{W}_{0}^{1, p}\left(\mathbb{R}^{3}\right)$ and $\frac{\partial}{\partial x_{1}} \mathcal{P}_{j} g \in L^{p}\left(\mathbb{R}^{3}\right)$ implies that $\mathcal{P}_{j} g \in L^{\frac{3 p}{3-2 p}}\left(\mathbb{R}^{3}\right)$ $($ see $[6])$.

(ii) Since $\frac{\partial g}{\partial x_{j}} \in L^{p}\left(\mathbb{R}^{n}\right)$, from Theorem 3.2, we have $J_{2} \frac{\partial g}{\partial x_{j}} \in W_{0}^{2, p}\left(\mathbb{R}^{n}\right)$. Proceeding as in the first part (i), we prove that

$$
\frac{\partial}{\partial x_{1}} J_{2}\left(\frac{\partial g}{\partial x_{j}}\right)=\frac{\partial}{\partial x_{j}} J_{2}\left(\frac{\partial g}{\partial x_{1}}\right) \text {. }
$$

Since $\frac{\partial g}{\partial x_{1}} \in W_{0}^{-1, p}\left(\mathbb{R}^{n}\right)$ with $p \geq n$, then we have $J_{2}\left(\frac{\partial g}{\partial x_{1}}\right) \in W_{0}^{1, p}\left(\mathbb{R}^{n}\right)$ and $\frac{\partial}{\partial x_{j}} J_{2}\left(\frac{\partial g}{\partial x_{1}}\right) \in$ $L^{p}\left(\mathbb{R}^{n}\right)$.

We now introduce the pair $\left(\boldsymbol{u}^{*}, \pi^{*}\right)$ defined by

$$
\begin{aligned}
& u_{i}^{*}=\mathcal{O}_{i j} f_{j}+\mathcal{P}_{i} g, \quad \pi^{*}=\mathcal{P}_{j} f_{j}+g-\mathcal{E} * \frac{\partial g}{\partial x_{1}} \quad \text { if } 1<p<n, \\
& u_{i}^{*}=\mathcal{O}_{i j} f_{j}-I_{2} \frac{\partial g}{\partial x_{i}}, \quad \pi^{*}=\mathcal{P}_{j}\left(f_{j}+G_{j}\right)+g \quad \text { if } p \geq n,
\end{aligned}
$$

where $\boldsymbol{G} \in \boldsymbol{L}^{p}\left(\mathbb{R}^{n}\right)$ is a (non unique) vector field such that $\operatorname{div} \boldsymbol{G}=\frac{\partial g}{\partial x_{1}}$. Next, for the case $n=3$, we introduce the notations used in [5] (see also [4]) for the resolution of the Oseen problem (4.15). Let $\gamma, \delta \in \mathbb{R}$ be such that $\gamma \in[3,4], \gamma>p, \delta \in\left[\frac{3}{2}, 2\right], \delta>p$. we define two real numbers $r=r(p, \gamma), s=s(p, \delta)$ as follow:

$$
\frac{1}{r}=\frac{1}{p}-\frac{1}{\gamma} \text { and } \frac{1}{s}=\frac{1}{p}-\frac{1}{\delta}
$$

Finally, we also introduce the space of polynomials

$$
\mathcal{N}_{k}=\left\{(\boldsymbol{\lambda}, \mu) \in \mathbb{P}_{k} \times \mathbb{P}_{k-1}^{\Delta}, \quad-\Delta \boldsymbol{\lambda}+\frac{\partial \boldsymbol{\lambda}}{\partial x_{1}}+\nabla \mu=\mathbf{0}, \operatorname{div} \boldsymbol{\lambda}=0\right\} .
$$

Combining Theorem 2.6 of , Proposition 4.1, Theorem 4.2 and Lemma 4.3, and results obtained in [2] for the case $n=2$ and in [5] for the case $n=3$, we easily prove the following result which gives an explicit form for the solutions of the Oseen equations for $\boldsymbol{f} \in \boldsymbol{L}^{p}\left(\mathbb{R}^{n}\right)$. 
Theorem 4.4. Let $(\boldsymbol{f}, g) \in \boldsymbol{L}^{p}\left(\mathbb{R}^{n}\right) \times Y_{0}^{1, p}\left(\mathbb{R}^{n}\right)$. Then the Oseen problem (4.15) has at least one solution $(\boldsymbol{u}, \pi) \in \boldsymbol{Z}_{0}^{2, p}\left(\mathbb{R}^{n}\right) \times W_{0}^{1, p}\left(\mathbb{R}^{n}\right)$ defined by

$$
\boldsymbol{u}=\boldsymbol{u}^{*}+\boldsymbol{\lambda}, \quad p=p^{*}+\mu,
$$

where $\left(\boldsymbol{u}^{*}, p^{*}\right)$ is given by $(4.30),(\boldsymbol{\lambda}, \mu) \in \mathcal{N}_{[2-n / p]}$ and we have the estimate

$$
\left\|\frac{\partial^{2} \boldsymbol{u}}{\partial x_{i} \partial x_{j}}\right\|_{\boldsymbol{L}^{p}\left(\mathbb{R}^{n}\right)}+\left\|\frac{\partial \boldsymbol{u}}{\partial x_{1}}\right\|_{\boldsymbol{L}^{p}\left(\mathbb{R}^{n}\right)}+\|\nabla p\|_{\boldsymbol{L}^{p}\left(\mathbb{R}^{n}\right)} \leq C\left(\|\boldsymbol{f}\|_{\boldsymbol{L}^{p}\left(\mathbb{R}^{n}\right)}+\|g\|_{Y_{0}^{1, p}\left(\mathbb{R}^{n}\right)}\right) .
$$

Additionally,

- If $n=2$, then we have

1) if $1<p<3$, then $\frac{\partial \boldsymbol{u}^{*}}{\partial x_{2}} \in \boldsymbol{L}^{\frac{3 p}{3-p}}\left(\mathbb{R}^{2}\right)$, in particular

i) when $1<p<2$, then $\frac{\partial \boldsymbol{u}^{*}}{\partial x_{2}} \in \boldsymbol{L}^{\frac{2 p}{2-p}}\left(\mathbb{R}^{2}\right)$ and satisfies

$$
\left\|\frac{\partial \boldsymbol{u}^{*}}{\partial x_{2}}\right\|_{\boldsymbol{L}^{3-p}\left(\mathbb{R}^{2}\right)}+\left\|\frac{\partial \boldsymbol{u}^{*}}{\partial x_{2}}\right\|_{\boldsymbol{L}^{\frac{2 p}{2-p}\left(\mathbb{R}^{2}\right)}} \leq C\left(\|\boldsymbol{f}\|_{\boldsymbol{L}^{p}\left(\mathbb{R}^{2}\right)}+\|g\|_{Y_{0}^{1, p}\left(\mathbb{R}^{2}\right)}\right) .
$$

ii) When $p=2$, then $\frac{\partial \boldsymbol{u}^{*}}{\partial x_{2}} \in \boldsymbol{L}^{q}\left(\mathbb{R}^{2}\right)$, for any $q \geq 6$ and

$$
\left\|\frac{\partial \boldsymbol{u}^{*}}{\partial x_{2}}\right\|_{\boldsymbol{L}^{q}\left(\mathbb{R}^{2}\right)} \leq C\left(\|\boldsymbol{f}\|_{\boldsymbol{L}^{p}\left(\mathbb{R}^{2}\right)}+\|g\|_{Y_{0}^{1, p}\left(\mathbb{R}^{2}\right)}\right) .
$$

iii) Finally when $2<p<3$, then $\frac{\partial \boldsymbol{u}^{*}}{\partial x_{2}} \in \boldsymbol{L}^{\infty}\left(\mathbb{R}^{2}\right)$ and

$$
\left\|\frac{\partial \boldsymbol{u}^{*}}{\partial x_{2}}\right\|_{\boldsymbol{L}^{\frac{3 p}{3-p}\left(\mathbb{R}^{2}\right)}}+\left\|\frac{\partial \boldsymbol{u}^{*}}{\partial x_{2}}\right\|_{\boldsymbol{L}^{\infty}\left(\mathbb{R}^{2}\right)} \leq C\left(\|\boldsymbol{f}\|_{\boldsymbol{L}^{p}\left(\mathbb{R}^{2}\right)}+\|g\|_{Y_{0}^{1, p}\left(\mathbb{R}^{2}\right)}\right) .
$$

2) If $1<p<\frac{3}{2}$, then $\boldsymbol{u}^{*} \in \boldsymbol{L}^{\frac{3 p}{3-2 p}}\left(\mathbb{R}^{2}\right) \cap \boldsymbol{L}^{\infty}\left(\mathbb{R}^{2}\right)$ and the following estimate holds

$$
\left\|\boldsymbol{u}^{*}\right\|_{\boldsymbol{L}^{\frac{3 p}{3-2 p}\left(\mathbb{R}^{2}\right)}}+\|\boldsymbol{u}\|_{L^{\infty}\left(\mathbb{R}^{2}\right)} \leq C\left(\|\boldsymbol{f}\|_{\boldsymbol{L}^{p}\left(\mathbb{R}^{2}\right)}+\|g\|_{Y_{0}^{1, p}\left(\mathbb{R}^{2}\right)}\right) .
$$

- If $n=3$, then we have

1) If $1<p<\frac{3}{2}$, then $\boldsymbol{u}^{*} \in \boldsymbol{L}^{s}\left(\mathbb{R}^{3}\right)$ and

$$
\left\|\boldsymbol{u}^{*}\right\|_{\boldsymbol{L}^{s}\left(\mathbb{R}^{3}\right)} \leq C\left(\|\boldsymbol{f}\|_{\boldsymbol{L}^{p}\left(\mathbb{R}^{3}\right)}+\|g\|_{Y_{0}^{1, p}\left(\mathbb{R}^{3}\right)}\right) .
$$

2) If $\frac{3}{2}<p<3$, then $\nabla \boldsymbol{u}^{*} \in \boldsymbol{L}^{r}\left(\mathbb{R}^{3}\right)$ and satisfies

$$
\left\|\nabla \boldsymbol{u}^{*}\right\|_{\boldsymbol{L}^{r}\left(\mathbb{R}^{3}\right)} \leq C\left(\|\boldsymbol{f}\|_{\boldsymbol{L}^{p}\left(\mathbb{R}^{3}\right)}+\|g\|_{Y_{0}^{1, p}\left(\mathbb{R}^{3}\right)}\right) .
$$

We now extend the definition of $\mathcal{O} * \boldsymbol{f}$ in the case where $\boldsymbol{f} \in \boldsymbol{W}_{0}^{-1, p}\left(\mathbb{R}^{n}\right)$ by setting

$$
\forall \varphi \in \mathcal{D}\left(\mathbb{R}^{n}\right), \quad\langle\mathcal{O} * \boldsymbol{f}, \varphi\rangle=:\langle\boldsymbol{f}, \check{\mathcal{O}} * \varphi\rangle_{W_{0}^{-1, p}\left(\mathbb{R}^{n}\right) \times W_{0}^{1, p^{\prime}}\left(\mathbb{R}^{n}\right)}
$$

where $\check{\mathcal{O}}(\boldsymbol{x})=\mathcal{O}(-\boldsymbol{x})$. 
Lemma 4.5. Assume $1<p<\infty$ and $f \in W_{0}^{-1, p}\left(\mathbb{R}^{2}\right) \perp \mathbb{P}_{\left[1-2 / p^{\prime}\right]}$. Then $\mathcal{O}_{2 j} * f \in W^{1, p}\left(\mathbb{R}^{2}\right)$ and the following estimate holds

$$
\left\|\mathcal{O}_{2 j} * f\right\|_{W^{1, p}\left(\mathbb{R}^{2}\right)} \leq C\|f\|_{W_{0}^{-1, p}\left(\mathbb{R}^{2}\right)} .
$$

Assume now $1<p<3$, then $\mathcal{O}_{11} * f \in L^{\frac{3 p}{3-p}}\left(\mathbb{R}^{2}\right), \nabla \mathcal{O}_{11} * f \in L^{p}\left(\mathbb{R}^{2}\right), \frac{\partial \mathcal{O}_{11}}{\partial x_{1}} * f \in W_{0}^{-1, p}\left(\mathbb{R}^{2}\right)$ and satisfy:

$$
\left\|\mathcal{O}_{11} * f\right\|_{L^{\frac{3 p}{3-p}\left(\mathbb{R}^{2}\right)}}+\left\|\nabla \mathcal{O}_{11} * f\right\|_{L^{p}\left(\mathbb{R}^{2}\right)}+\left\|\frac{\partial \mathcal{O}_{11}}{\partial x_{1}} * f\right\|_{W_{0}^{-1, p}\left(\mathbb{R}^{2}\right)} \leq C\|f\|_{W_{0}^{-1, p}\left(\mathbb{R}^{2}\right)} .
$$

The proof this lemma can be found in [1].

Lemma 4.6. Assume $1<p<4$ and $\boldsymbol{f} \in \boldsymbol{W}_{0}^{-1, p}\left(\mathbb{R}^{3}\right) \perp \mathbb{P}_{\left[1-3 / p^{\prime}\right]}$. Then $\boldsymbol{O} * \boldsymbol{f} \in \boldsymbol{L}^{\frac{4 p}{4-p}}\left(\mathbb{R}^{3}\right)$, $\nabla \mathcal{O} \in \boldsymbol{L}^{p}\left(\mathbb{R}^{3}\right)$ and we have

$$
\|\mathcal{O} * \boldsymbol{f}\|_{L^{\frac{4 p}{4-p}\left(\mathbb{R}^{3}\right)}}+\|\nabla \mathcal{O} * \boldsymbol{f}\|_{\boldsymbol{L}^{p}\left(\mathbb{R}^{n}\right)} \leq C\|\boldsymbol{f}\|_{W_{0}^{-1, p}\left(\mathbb{R}^{3}\right)} .
$$

Moreover, the following assertions hold.

(i) If $1<p<3$, then $\mathcal{O} * \boldsymbol{f} \in \boldsymbol{L}^{\frac{3 p}{3-p}}\left(\mathbb{R}^{3}\right)$ and

$$
\|\mathcal{O} * \boldsymbol{f}\|_{L^{3-p}\left(\mathbb{R}^{3}\right)} \leq C\|\boldsymbol{f}\|_{W_{0}^{-1, p}\left(\mathbb{R}^{3}\right)} .
$$

(ii) If $p=3$, then $\mathcal{O} * \boldsymbol{f} \in \boldsymbol{L}^{q}\left(\mathbb{R}^{3}\right)$, for any $q \geq 12$.

(iii) If $3<p<4$, then $\mathcal{O} * \boldsymbol{f} \in \boldsymbol{L}^{\infty}\left(\mathbb{R}^{3}\right)$.

Proof. The above properties are proved for the fundamental solution $\mathcal{O}$ (see [1], Theorem 4.9 ) and the proof is similar for the Oseen fundamental solution $\mathcal{O}$.

Remark 4.7. For a given $f$ in $W_{0}^{-1, p}\left(\mathbb{R}^{2}\right) \perp \mathbb{P}_{\left[1-2 / p^{\prime}\right]}$, then $\mathcal{O}_{2 j} * f$ exists for any $1<p<\infty$, while $\mathcal{O}_{11} * f$ has no sense for $p \geq 3$.

For $p \geq 3$ and $f \in W_{0}^{-1, p}\left(\mathbb{R}^{2}\right)$, we now define the operator $\mathcal{O}_{11} f$ as follows:

$$
\mathcal{O}_{11} f(\boldsymbol{x})=\int_{\mathbb{R}^{2}}\left(\frac{\partial}{\partial x_{j}} \mathcal{O}_{11}(\boldsymbol{x}-\boldsymbol{y})-\frac{\partial}{\partial x_{j}} \mathcal{O}_{11}(-\boldsymbol{y})\right) F_{j}(\boldsymbol{y}) d \boldsymbol{y}
$$

where $\boldsymbol{F} \in \boldsymbol{L}^{p}\left(\mathbb{R}^{2}\right)$ is a vector field such that $f=\operatorname{div} \boldsymbol{F}$.

Similarly for $p \geq 4$ and $f \in W_{0}^{-1, p}\left(\mathbb{R}^{3}\right)$, we define the following operator $\mathcal{O}$ such for any $i, j=1,2,3$,

$$
\mathcal{O}_{i j} f(\boldsymbol{x})=\int_{\mathbb{R}^{3}}\left(\frac{\partial}{\partial x_{k}} \mathcal{O}_{i j}(\boldsymbol{x}-\boldsymbol{y})-\frac{\partial}{\partial x_{k}} \mathcal{O}_{i j}(-\boldsymbol{y})\right) F_{k}(\boldsymbol{y}) d \boldsymbol{y},
$$

where $\boldsymbol{F} \in \boldsymbol{L}^{p}\left(\mathbb{R}^{n}\right)$ is a vector field such that $f=\operatorname{div} \boldsymbol{F}$. Thanks to Theorem 4.2, we have the following result. 
Theorem 4.8. i) Assume $n=2$, then for any $p \geq 3$, the operator

$$
\mathcal{O}_{11}: W_{0}^{-1, p}\left(\mathbb{R}^{2}\right) \mapsto W_{0}^{1, p}\left(\mathbb{R}^{2}\right)
$$

is continuous. In addition, we have

$$
\nabla \mathcal{O}_{11} f=\nabla \frac{\partial \mathcal{O}_{11}}{\partial x_{j}} * F_{j}
$$

ii) Assume $n=3$, then the operator

$$
\mathcal{O}: W_{0}^{-1, p}\left(\mathbb{R}^{3}\right) \mapsto W_{0}^{1, p}\left(\mathbb{R}^{3}\right)
$$

is continuous if $p \geq 4$.

Before stating our last result, we need two preliminary lemmas that take into account the second equation of (4.15).

Lemma 4.9. Assume $g \in W_{0}^{-2, p}\left(\mathbb{R}^{n}\right) \perp \mathbb{P}_{\left[2-n / p^{\prime}\right]}$. Then $\mathcal{E} * g \in L^{p}\left(\mathbb{R}^{n}\right)$ and we have

$$
\|\mathcal{E} * g\|_{L^{p}\left(\mathbb{R}^{n}\right)} \leq C\|g\|_{W_{0}^{-2, p}\left(\mathbb{R}^{n}\right)} .
$$

Proof. For any $\varphi \in \mathcal{D}\left(\mathbb{R}^{n}\right)$, we have

$$
\langle\mathcal{E} * g, \varphi\rangle_{\mathcal{D}^{\prime}\left(\mathbb{R}^{n}\right) \times \mathcal{D}\left(\mathbb{R}^{n}\right)}=\langle g, \mathcal{E} * \varphi\rangle_{W_{0}^{-2, p}\left(\mathbb{R}^{n}\right) \times W_{0}^{2, p^{\prime}}\left(\mathbb{R}^{n}\right)} .
$$

Next, since $g \in W_{0}^{-2, p}\left(\mathbb{R}^{n}\right) \perp \mathbb{P}_{\left[2-n / p^{\prime}\right]}$, for any $\lambda \in \mathbb{P}_{\left[2-n / p^{\prime}\right]}$, we have

$$
\langle g, \mathcal{E} * \varphi\rangle_{W_{0}^{-2, p}\left(\mathbb{R}^{n}\right) \times W_{0}^{2, p^{\prime}}\left(\mathbb{R}^{n}\right)}=\langle g, \mathcal{E} * \varphi+\lambda\rangle_{W_{0}^{-2, p}\left(\mathbb{R}^{n}\right)} .
$$

It follows that

$$
\left|\langle g, \mathcal{E} * \varphi\rangle_{W_{0}^{-2, p}\left(\mathbb{R}^{n}\right) \times W_{0}^{2, p^{\prime}}\left(\mathbb{R}^{n}\right)}\right| \leq C\|g\|_{W_{0}^{-2, p}\left(\mathbb{R}^{n}\right)} \inf _{\lambda \in \mathbb{P}_{\left[2-n / p^{\prime}\right]}}\|\mathcal{E} * \varphi+\lambda\|_{W_{0}^{2, p^{\prime}}\left(\mathbb{R}^{n}\right)} .
$$

Using now (2.2) and the Calderón-Zygmund inequality we can write

$$
\begin{aligned}
\left|\langle g, \mathcal{E} * \varphi\rangle_{\mathcal{D}^{\prime}\left(\mathbb{R}^{n}\right) \times \mathcal{D}\left(\mathbb{R}^{n}\right)}\right| & \leq C\|g\|_{W_{0}^{-2, p}\left(\mathbb{R}^{n}\right)}\left\|\frac{\partial^{2}(\mathcal{E} * \varphi)}{\partial x_{i} \partial x_{j}}\right\|_{L^{p^{\prime}\left(\mathbb{R}^{n}\right)}} \\
& \leq C\|g\|_{W_{0}^{-2, p}\left(\mathbb{R}^{n}\right)}\|\Delta(\mathcal{E} * \varphi)\|_{L^{p^{\prime}\left(\mathbb{R}^{n}\right)}} \\
& \leq C\|g\|_{W_{0}^{-2, p}\left(\mathbb{R}^{n}\right)}\|\| \varphi \|_{L^{p^{\prime}\left(\mathbb{R}^{n}\right)}},
\end{aligned}
$$

which ends the proof.

Lemma 4.10. Assume $1<p<\infty$ and $g \in X_{p}\left(\mathbb{R}^{n}\right)$ such that

$$
\forall \lambda \in \mathbb{P}_{\left[2-n / p^{\prime}\right]}, \quad\left\langle\frac{\partial g}{\partial x_{1}}, \lambda\right\rangle_{W_{0}^{-2, p}\left(\mathbb{R}^{n}\right) \times W_{0}^{2, p^{\prime}}\left(\mathbb{R}^{n}\right)}=0 .
$$

Then $\mathcal{P}_{j} g \in Y_{0}^{1, p}\left(\mathbb{R}^{n}\right)$ and there exists $C>0$ such that

$$
\left\|\mathcal{P}_{j} g\right\|_{Y_{0}^{1, p}\left(\mathbb{R}^{n}\right)} \leq C\|g\|_{X_{p}\left(\mathbb{R}^{n}\right)} .
$$


Proof. Since $g \in X_{p}\left(\mathbb{R}^{n}\right)$, then from Theorem 3.1, $\mathcal{P}_{j} g \in W_{0}^{1, p}\left(\mathbb{R}^{n}\right)$ and we have

$$
\left\|\mathcal{P}_{j} g\right\|_{W_{0}^{1, p}\left(\mathbb{R}^{n}\right)} \leq C\|g\|_{L^{p}\left(\mathbb{R}^{n}\right)} .
$$

It remains to prove that $\frac{\partial}{\partial x_{1}} \mathcal{P}_{j} g \in W_{0}^{-1, p}\left(\mathbb{R}^{n}\right)$. For any $\varphi \in \mathcal{D}\left(\mathbb{R}^{n}\right)$, we have

$$
\left|\left\langle\frac{\partial}{\partial x_{1}} \mathcal{P}_{j} g, \varphi\right\rangle_{\mathcal{D}^{\prime}\left(\mathbb{R}^{n}\right) \times \mathcal{D}\left(\mathbb{R}^{n}\right)}\right|=\left|\left\langle g, \frac{\partial}{\partial x_{1}}\left(\check{\mathcal{P}}_{j} * \varphi\right)\right\rangle_{L^{p}\left(\mathbb{R}^{n}\right) \times L^{p^{\prime}\left(\mathbb{R}^{n}\right)}}\right|,
$$

where $\check{\mathcal{P}}_{j}(\boldsymbol{x})=\mathcal{P}_{j}(-\boldsymbol{x})$. It follows that

$$
\begin{aligned}
\left|\left\langle\frac{\partial}{\partial x_{1}} \mathcal{P}_{j} g, \varphi\right\rangle_{\mathcal{D}^{\prime}\left(\mathbb{R}^{n}\right) \times \mathcal{D}\left(\mathbb{R}^{n}\right)}\right| & =\left|\left\langle\frac{\partial g}{\partial x_{1}}, \mathcal{E} * \frac{\partial \varphi}{\partial x_{j}}\right\rangle_{W_{0}^{-2, p}\left(\mathbb{R}^{n}\right) \times W_{0}^{2, p^{\prime}}\left(\mathbb{R}^{n}\right)}\right| \\
& =\left|\left\langle\frac{\partial g}{\partial x_{1}} * \mathcal{E}, \frac{\partial \varphi}{\partial x_{j}}\right\rangle_{L^{p}\left(\mathbb{R}^{n}\right) \times L^{p^{\prime}}\left(\mathbb{R}^{n}\right)}\right|
\end{aligned}
$$

From Lemma 4.9, we get

$$
\left|\left\langle\frac{\partial}{\partial x_{1}} \mathcal{P}_{j} g, \varphi\right\rangle_{\mathcal{D}^{\prime}\left(\mathbb{R}^{n}\right) \times \mathcal{D}\left(\mathbb{R}^{n}\right)}\right| \leq C\left\|\frac{\partial g}{\partial x_{1}}\right\|_{W_{0}^{-2, p}\left(\mathbb{R}^{n}\right)}\|\varphi\|_{W_{0}^{1, p^{\prime}\left(\mathbb{R}^{n}\right)}} .
$$

Thus we deduce that $\frac{\partial}{\partial x_{1}} \mathcal{P}_{j} g \in W_{0}^{-1, p}\left(\mathbb{R}^{3}\right)$ and

$$
\left\|\frac{\partial}{\partial x_{1}} \mathcal{P}_{j} g\right\|_{W_{0}^{-1, p}\left(\mathbb{R}^{n}\right)} \leq C\left\|\frac{\partial g}{\partial x_{1}}\right\|_{W_{0}^{-2, p}\left(\mathbb{R}^{n}\right)} .
$$

For $\boldsymbol{f} \in W_{0}^{-1, p}\left(\mathbb{R}^{n}\right) \perp \mathbb{P}_{\left[1-n / p^{\prime}\right]}$ and $g \in X_{p}\left(\mathbb{R}^{n}\right)$, we now define the pair $\left(\boldsymbol{u}^{*}, p^{*}\right)$

$$
\begin{aligned}
u_{i}^{*} & =\mathcal{O}_{i j} * f_{j}+\mathcal{P}_{j} g \quad \text { if } 1<p<n, \quad u_{i}^{*}=\mathcal{O}_{i j} f_{j}+\mathcal{P}_{j} g \text { if } p \geq n, \\
p^{*} & =-\mathcal{E} *\left(\operatorname{div} \boldsymbol{f}+\frac{\partial g}{\partial x_{1}}\right)+g
\end{aligned}
$$

Combining results of [2] for the case $n=2$, [5] for the case $n=3$, Lemma 4.6, Theorem 4.8 and the previous lemmas, we easily prove the following result.

Theorem 4.11. Let $(\boldsymbol{f}, g) \in \boldsymbol{W}_{0}^{-1, p}\left(\mathbb{R}^{n}\right) \times X_{p}\left(\mathbb{R}^{n}\right)$ satisfy the following compatibility conditions

$$
\forall \boldsymbol{\lambda} \in \mathbb{P}_{\left[1-n / p^{\prime}\right]}, \quad\langle\boldsymbol{f}, \boldsymbol{\lambda}\rangle_{\boldsymbol{W}_{0}^{-1, p}\left(\mathbb{R}^{n}\right) \times \boldsymbol{W}_{0}^{1, p^{\prime}}\left(\mathbb{R}^{n}\right)}=0
$$

and

$$
\forall \lambda \in \mathbb{P}_{\left[2-n / p^{\prime}\right]}, \quad\left\langle\frac{\partial g}{\partial x_{1}}, \lambda\right\rangle_{W_{0}^{-2, p}\left(\mathbb{R}^{n}\right) \times W_{0}^{2, p^{\prime}}\left(\mathbb{R}^{n}\right)}=0 .
$$

Then the Oseen problem (4.15) has at least a solution $(\boldsymbol{u}, p) \in Y_{0}^{1, p}\left(\mathbb{R}^{n}\right) \times L^{p}\left(\mathbb{R}^{n}\right)$ defined by

$$
\boldsymbol{u}=\boldsymbol{u}^{*}+\lambda, \quad p=p^{*}
$$


where the pair $\left(\boldsymbol{u}^{*}, p^{*}\right)$ is given by (4.34) and $\boldsymbol{\lambda} \in \mathbb{P}_{[1-n / p]}$. The following estimate holds

$$
\inf _{\boldsymbol{\lambda} \in \mathbb{P}_{[1-n / p]}}\|\boldsymbol{u}+\boldsymbol{\lambda}\|_{\mathbf{Y}_{0}^{1, p}\left(\mathbb{R}^{n}\right)}+\left\|p^{*}\right\|_{\boldsymbol{L}^{p}\left(\mathbb{R}^{n}\right)} \leq C\left(\|\boldsymbol{f}\|_{\boldsymbol{W}_{0}^{-1, p}\left(\mathbb{R}^{n}\right)}+\|g\|_{X_{p}\left(\mathbb{R}^{n}\right)}\right) .
$$

Moreover, if $n=3$ and $1<p<3$, then $\boldsymbol{u}^{*} \in \boldsymbol{L}^{r}\left(\mathbb{R}^{3}\right)$ and we have

$$
\left\|\boldsymbol{u}^{*}\right\|_{\boldsymbol{L}^{r}\left(\mathbb{R}^{3}\right)} \leq C\left(\|\boldsymbol{f}\|_{\boldsymbol{W}_{0}^{-1, p}\left(\mathbb{R}^{3}\right)}+\|g\|_{X_{p}\left(\mathbb{R}^{3}\right)}\right) .
$$

\section{Acknowledgement}

We would like to thank Pierre-Gilles Lemarié Rieusset and Yves Meyer for many helpful comments and discussions.

\section{References}

[1] Amrouche, C., Bouzit, H.: $\mathrm{L}^{p}$-inequalities for scalar Oseen potential. J. Math. Anal. Appl. 337, 753-770 (2008).

[2] Amrouche, C., Bouzit, H.: Two dimensional strong and generalized solutions for the stationary Oseen equations. Submitted.

[3] Amrouche, C., Girault, V., Giroire, J.: Weighted Sobolev spaces for Laplace's equation in $\mathbb{R}^{n}$. J. Math. Pures Appl. (9) 73(6), 579-606 (1994).

[4] Amrouche, C., Razafison, U.: Weighted estimates for the Oseen problem in $\mathbb{R}^{3}$. Appl. Math. Lett. 19(1), 56-62 (2006).

[5] Amrouche, C., Razafison, U.: The stationary Oseen equations in $\mathbb{R}^{3}$. An approach in weighted Sobolev spaces. J. Math. Fluid Mech. 9, 211-225 (2007).

[6] Babenko, K.I.: On stationary solutions of the problem of flow past a body of a viscous incompressible fluid. Math. USSR, Sb. 20, 371-401 (2005).

[7] Boulmezaoud, T.Z., Razafison, U.: On the steady Oseen problem in the whole space. Hiroshima Math. J. 35(3), 371-401 (2005).

[8] Farwig, R.: The stationary exterior 3D-problem of Oseen and Navier-Stokes equations in anisotropically weighted Sobolev spaces. Math. Z. 211(3), 409-447 (1992).

[9] Farwig, R., Sohr, H.: Weighted estimates for the Oseen equations and the NavierStokes equations in exterior domains. In Theory of the Navier-Stokes equations, volume 47 of Ser. Adv. Math. Appl. Sci., 11-30. World Sci. Publishings, River Edge, NJ, (1998). 
[10] Oseen, C. W.: Neuere Methoden und Ergebnisse in der Hydrodynamik. Leipzig, Akadem. Verlagsgesellschaft M. B. H, Leipzig (1927).

[11] Razafison, U.: Anisotropic weighted $L^{p}$ spaces for the stationary exterior 3D-problem of Oseen. J. Math. Anal. Appl. 323(1), 275-292 (2006).

[12] Stein, E.M.: Singular integrals and differentiability properties of functions. Princeton University Press, Princeton, New Jersey (1970). 